\title{
Model-based Control Design of a Diesel Oxidation Catalyst
}

\author{
Olivier Lepreux* Yann Creff* Nicolas Petit ** \\ * IFP Lyon, Technology, Computer Science and Applied Mathematics \\ Division, BP 3, 69360 Solaize, France (e-mail: olivier.lepreux @ ifp.fr, \\ yann.creff@ifp.fr) \\ ** Centre Automatique et Systèmes, Mathématiques et Systèmes, MINES \\ ParisTech, 60, bd. Saint-Michel, 75272 Paris Cedex 06, France (e-mail: \\ nicolas.petit@mines-paristech.fr)
}

\begin{abstract}
:
This paper proposes a control strategy for a Diesel Oxidation Catalyst (DOC) which is grounded on a one-dimensional distributed parameter model. This first principles model for the propagation of the temperature variations accounts for spatially distributed heat generation (due to oxidation of reductants). As is discussed, heat generation can be regarded as equivalent inlet temperature variations. This fact is supported by experimental results. By nature, DOC outlet temperature response includes long and timevarying delays. An approximation of the proposed model allows to derive delays analytically, and can be used to schedule control parameters. As a consequence, it is easy to design several standard controllers for the DOC outlet temperature which account for the effects of the inlet temperature (disturbance) and the reductant (control). In this paper, simulation results are presented for a PI, a PID, and a Smith predictor. Interestingly, the three controllers use solely parameters determined from the previous analysis and do not need any extra tuning parameter. The strategies are tested on a standard NEDC driving cycle in simulation. It appears that, among these standard strategies, the DOC partial derivative equations can be efficiently controlled using the presented Smith predictor.
\end{abstract}

Keywords: Automotive exhaust aftertreatment systems, Diesel oxidation catalyst, Distributed-parameter systems, Boundary control, Control applications

\section{INTRODUCTION}

\subsection{Motivation}

On most new Diesel vehicles, increasing requirements regarding particulate matter emissions (Ecopoint Inc., 2008) are satisfied using a Diesel Particulate Filter (DPF). This filter, located in the vehicle exhaust line, stores particulate matter until it is burnt in an active regeneration process (Bisset, 1984). During this phase, DPFs behave like potentially unstable reactors (Achour, 2001), and their inlet temperature must be carefully controlled to prevent filter runaway.

In most current aftertreatment architectures (Koltsakis and Stamatelos, 1997), a Diesel Oxidation Catalyst (DOC) is placed upstream the DPF in the vehicle exhaust line. To increase the DPF inlet temperature, reductant is oxidized in the DOC, which, in turn, increases its outlet temperature. The DOC also conveys, up to some heat losses, its inlet enthalpy flow: in other words, inlet temperature variations propagate through the DOC.

A DOC is a chemical system difficult to control. Classical models are usually composed of a dozen of coupled partial differential equations (PDEs) (Depcik and Assanis, 2005), which complexify the development of model-based control laws. Experimentally, it can be observed that a step change on the inlet temperature propagates to the output of the system with long response times (Oh and Cavendish, 1982). Depending on the engine outlet gas flow rate, these response times significantly vary: they roughly decrease by a factor of 10 from idle speed to full load. Strategies that are commonly used to deal with this problem rely on look-up tables, which, in practice, are difficult and time-consuming to calibrate.

The purpose of this paper is to propose implementable control laws tuned according to a simple control-oriented model. This approach allows faster calibration. To achieve this goal, simplification of the above-mentioned classical models is needed.

After a presentation of a mathematical formulation of the control problem in the second part of this introduction, we show in section 2 how the model proposed in Lepreux et al. (2008), initially using inlet temperature as control variable, can be used to accurately describe actual cases of engineering interest, i.e. cases where the reductant flow is the control variable. Then, we show in section 3 how to approximate the model. Finally, in section 4, this approximation is used to tune several classic controllers. Simulation results serve as comparisons and stress that a Smith predictor tuned using the proposed methodology represents an efficient controller for the DOC.

\subsection{Problem Formulation}

It has been shown in Lepreux et al. (2008) that, considering only inlet temperature variations and neglecting chemical reactions, a DOC thermal behavior can be accurately described by the following model 


$$
\left\{\begin{aligned}
\frac{\partial T}{\partial t}(z, t)+v \frac{\partial T}{\partial z}(z, t) & =-k_{1}\left(T(z, t)-T_{s}(z, t)\right) \\
\frac{\partial T_{s}}{\partial t}(z, t) & =k_{2}\left(T(z, t)-T_{s}(z, t)\right)
\end{aligned}\right.
$$

with boundary control

$$
T(z=0, t)=T^{i n}(t)
$$

where $T$ and $T_{s}$ are respectively gas and solid temperature variations about steady state, $v$ is the channel gas speed which can be derived from mass flow, parameters $\left(k_{1}, k_{2}\right)$ can be either derived from usual correlation (Osizik, 1977) or identified from experimental data (Lepreux et al., 2008). The output of the system is the outlet gas temperature

$$
T^{\text {out }}(t)=T(z=L, t)
$$

Considering steady-state initial conditions

$$
\left\{\begin{array}{l}
T(z, 0)=0 \\
T_{s}(z, 0)=0
\end{array}\right.
$$

system (1) yields the transfer function

$$
\hat{T}(z, s)=\hat{T}^{i n}(s) \exp \left(-\frac{z}{v} s-\frac{k_{1} z}{v}+\frac{m(z)}{s+k_{2}}\right)
$$

where $m(z)=k_{1} k_{2} z / v, \hat{x}$ is the Laplace transform of $x$, and $s$ is the Laplace variable. We note $\Upsilon$ the Heaviside function. The system step response is

$$
\begin{aligned}
& T(z, t)=\Upsilon\left(t-\frac{z}{v}\right) \exp \left(-\frac{k_{1} z}{v}\right) \times \\
& {\left[1+\int_{0}^{t-z / v} \exp \left(-k_{2} \tau\right) \sqrt{\frac{m(z)}{\tau}} \mathrm{I}_{1}(2 \sqrt{m(z) \tau}) d \tau\right]}
\end{aligned}
$$

For an easy evaluation of (3), a formulation using power series expansion is given in Lepreux et al. (2008).

\section{REDUCTANT FLOW AS CONTROL VARIABLE}

It is shown in Lepreux et al. (2008) that experimentally measured step responses of the system can be identified to model (1) with good quality. However, this representation might seem a bit simplistic in view of real applications since inlet temperature variations are difficult to control and cannot be used directly as control variable. In practice, reductants (hydrocarbons $H C$ ) are injected at the inlet of the DOC. They are oxidized on the catalyst and, consequently, increases the DOC temperature. In this section, we compute $H C$ step response and compare it against $T^{i n}$ step response.

\subsection{Model with Heat Source}

During the regeneration process, the DOC is working at high temperatures, which ensures that the rate of conversion of reductants is high. Moreover, large quantity of $H C$ is injected to generate exothermicity. Consequently, the inlet fraction of this reductant is very important, and its effect is dominating over other species'. By construction, a DOC is designed to yield large heat and mass transfer. These transfers are very effective, and the time scales implying the thermal phenomena are much lower than the ones implying chemical reactions. For the experiments presented in $\S 2.3$ gas flows through the DOC approximatively 1000 times faster than the outlet temperature response time. For these reasons, to model the DOC thermal behavior, we propose to encompass all the chemical reactions in a "source term $\Psi$ ", leading to the following model

$$
\left\{\begin{array}{l}
\frac{\partial T}{\partial t}(z, t)+v \frac{\partial T}{\partial z}(z, t)=-k_{1}\left(T(z, t)-T_{s}(z, t)\right) \\
\frac{\partial T_{s}}{\partial t}(z, t)=k_{2}\left(T(z, t)-T_{s}(z, t)\right)+\Psi(z, t)
\end{array}\right.
$$

where $\Psi(z, t)$ is the control variable and $T^{i n}(t)$ is regarded as a disturbance. $\Psi$ includes the sum of the enthalpies of the various reactions taking place inside the DOC. We formulate a strong simplifying assumption. Namely, we assume that the rate of reaction is independent of the species concentration. Further, we also assume that it is independent of the temperature. In other words, $\Psi$ is constant over some spatial interval. These assumptions are supported by experimental identification results of $\S 2.3$. Over the whole range of considered operating conditions, the obtained results are quite accurate. We note $L_{c}$ the length of the portion of the DOC where the enthalpy of reaction is released (see Fig. 1). Formally, we consider the

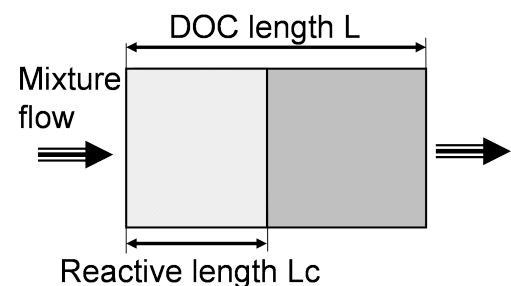

Fig. 1. HC reaction zone

following discontinuous function

$$
\left\{\begin{array}{l}
\hat{\Psi}(z, s)=\alpha / s, \quad 0 \leq z \leq L_{c} \\
\hat{\Psi}(z, s)=0, \quad L_{c}<z \leq L
\end{array}\right.
$$

Then, several steps of operational calculus on (4) lead to

$\hat{T}\left(L_{c}, s\right)=\hat{T}^{i n} \exp \left(-\hat{A}(s) L_{c}\right)+\frac{\hat{B}(s)}{\hat{A}(s)}\left(1-\exp \left(-\hat{A}(s) L_{c}\right)\right)$

with

$$
\left\{\begin{array}{l}
\hat{A}(s)=\frac{1}{v}\left(s+k_{1}-\frac{k_{1} k_{2}}{s+k_{2}}\right) \\
\hat{B}(s)=\frac{k_{1}}{v} \frac{\alpha}{s\left(s+k_{2}\right)}
\end{array}\right.
$$

In (6), the first term corresponds to the transfer from the inlet temperature $T^{i n}(t)$ to the output $T\left(L_{c}, t\right)$, while the second term corresponds to the transfer from the input signal $\hat{\Psi}$ defined in (5) to the output $T\left(L_{c}, t\right)$. The linearity of the two effects will be used to study these phenomena separately in our control strategy. Further, for $z>L_{c}$, equation (4) gives

$$
\hat{T}(z, s)=\hat{T}\left(L_{c}, s\right) \exp \left(-\hat{A}\left(z-L_{c}\right)\right)
$$

and, we get

$$
\begin{gathered}
\hat{T}(z, s)=\hat{T}^{i n} \exp (-\hat{A} z)-\frac{\hat{B}}{\hat{A}} \exp (-\hat{A} z) \\
+\frac{\hat{B}}{\hat{A}} \exp \left(-\hat{A}\left(z-L_{c}\right)\right)
\end{gathered}
$$

Eventually, by an inverse Laplace transform of (7) (Abramowitz and Stegun, 1965) (Angot, 1982) (Wolfram Research Inc., 2006), one obtains the reductant step response (8) 


$$
\left\{\begin{array}{l}
T(z, t)=\Upsilon(t-z / v) \exp \left(-\frac{k_{1} z}{v}\right) M(z, t-z / v) \\
-\Upsilon(t-z / v) \exp \left(-\frac{k_{1} z}{v}\right) F(z, t-z / v) \\
\quad \Upsilon(t-z / v) \exp \left(-\frac{k_{1} z}{v}\right) F\left(z-L_{c}, t-z / v\right) \\
M(z, t)=T^{i n}(t) \\
\quad+\int_{0}^{t} \exp \left(-k_{2} \tau\right) \sqrt{\frac{m(z)}{\tau}} \mathrm{I}_{1}(2 \sqrt{m(z) \tau}) T^{i n}(t-\tau) d \tau \\
F(z, t)=g(t) \\
+\int_{0}^{t} \exp \left(-k_{2} \tau\right) \sqrt{\frac{m(z)}{\tau}} \mathrm{I}_{1}(2 \sqrt{m(z) \tau}) g(t-\tau) d \tau \\
g(t)=\frac{k_{1} \alpha}{k_{1}+k_{2}} t-\frac{k_{1} \alpha}{\left(k_{1}+k_{2}\right)^{2}}\left(1-\exp \left(-\left(k_{1}+k_{2}\right) t\right)\right)
\end{array}\right.
$$

\subsection{Fitting the heat source model with an equivalent no-source model}

The static gain $G_{T^{i n}}$ of the transfer from the inlet temperature $T^{i n}$ to the output $T(z, t)$ is equal to 1 (Lepreux et al., 2008). The static gain $G_{u}$ of the transfer from the input signal $\hat{\Psi}$ to the output $T(z, t)$ can be computed using (7)

$$
\begin{aligned}
G_{u} & =\lim _{t \rightarrow \infty} T(t)=\lim _{s \rightarrow 0} s \hat{T}(s) \\
= & \lim _{s \rightarrow 0}\left[s \frac{\hat{B}}{\hat{A}}\left(1-\hat{A}\left(z-L_{c}\right)\right)-s \frac{\hat{B}}{\hat{A}}(1-\hat{A} z)\right] \\
= & \frac{k_{1} \alpha L_{c}}{k_{2} v}
\end{aligned}
$$

These last formulas are used during the identification and normalization process in this Section and for controllers design in Section 4. In practice, it is possible to relate $\alpha, L_{c}$, the current $H C$ conversion efficiency and the amount of injected reductants (which is itself related to the injector energizing time): for a given (identified) $L_{c}, \alpha$ can be regarded as a control variable.

In Fig. 2a, it is shown that the overall shape of the reductant step response, computed with (8), is very similar to the $T^{i n}$ step response (3). This similarity suggests that it is possible to approximate $H C$ step response by $T^{i n}$ step response at the expense of an additional identification procedure. We show in Fig. $2 b$ that it suffices to adapt the DOC length, using the model with no source (1), to get results very close to the ones obtained with the model with source $(4)^{1}$. In other words, generating enthalpy by $H C$ is quite equivalent to generating enthalpy by $T^{i n}$ with a DOC having a shorter length. Hence, the temperature response of the DOC associated to the $T^{i n}$ variations are slower than those associated to $H C$. From a control point of view, this allows us to reject the $T^{i n}$ disturbance.

\subsection{Experimental model validation}

As we stressed it in the previous discussion, considering an additional model adaptation of parameters $\left(L_{c}\right.$ is a piecewise linear function of $v$ ), model (1) and model (4) yield pretty similar results. In Fig. 3, we present experimental $H C$ step responses under various operating conditions. To obtain these data, a 2.2-L 4-cylinder Diesel engine equipped with a 3inch long 5.66-inch diameter 400-cpsi DOC was tested. These

\footnotetext{
1 further details of this adaptation will be treated in a forthcoming publication
}
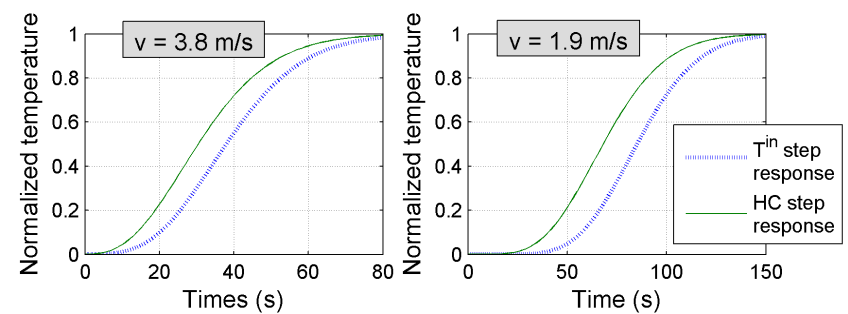

(a) Comparison between $H C$ step response and $T^{i n}$ step response. Analytic results obtained respectively from (8) and (3).
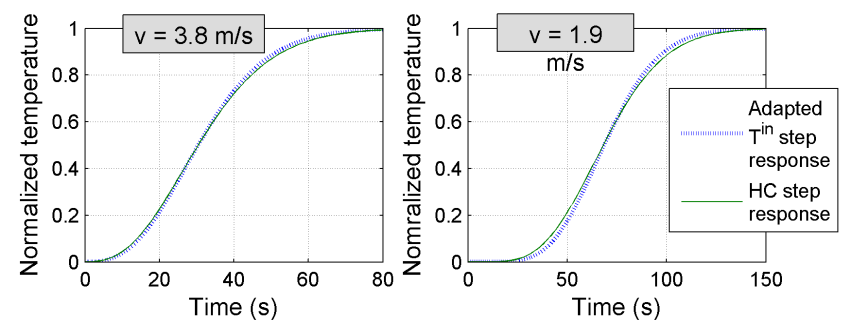

(b) Comparison between $H C$ step response (model with source) and adapted $T^{i n}$ step response (model with no source)

Fig. 2. $H C$ step response approximation in various operating conditions

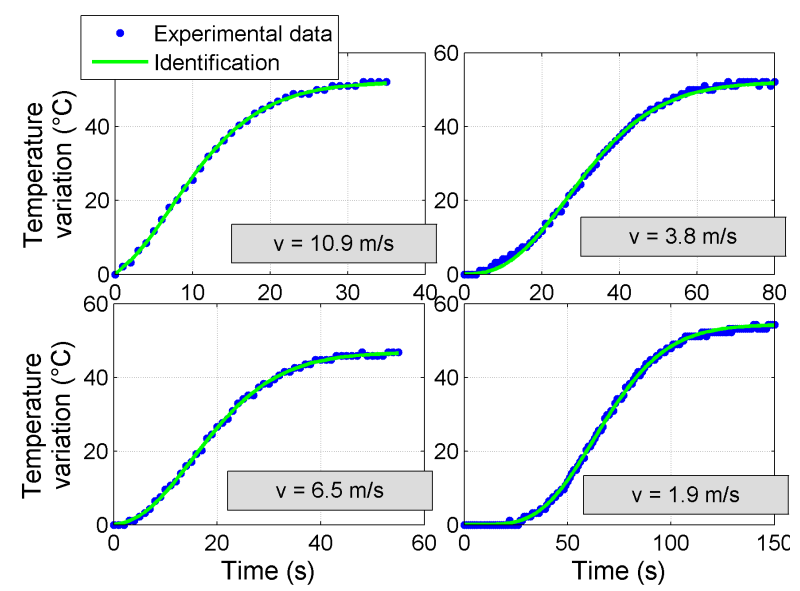

Fig. 3. Experimental $H C$ step response identified to $T^{i n}$ model (1) in various operating conditions

responses are well represented by the equivalent $T^{\text {in }}$ step response (3) corresponding to the model with no source (1). It is shown that the model with no source kindly fits experimental data, usually described using a source term.

\section{APPROXIMATING DOC EQUATIONS}

We wish to simplify the previous model further. The desired representation is a first order plus delay model, which belongs to a class of models relatively easy to design a controller for (Silva et al., 2005).

For small values of $|s|$ (i.e. the range of low frequencies), the DOC transfer function (2) can be approximated in the following way 


$$
\begin{aligned}
& \exp \left(\frac{m(z)}{s+k_{2}}\right) \\
& =\exp \left(-(1-\nu) \frac{k_{1} z}{k_{2} v} s\right) \exp \left(\frac{k_{1} z}{v}\left((1-\nu) s / k_{2}+\frac{1}{1+s / k_{2}}\right)\right) \\
& \approx \exp \left(-(1-\nu) \frac{k_{1} z}{k_{2} v} s\right) \exp \left((1-\nu) s / k_{2}+1-s / k_{2}\right)^{\frac{k_{1} z}{v}} \\
& \approx \exp \left(-(1-\nu) \frac{k_{1} z}{k_{2} v} s\right) \exp \left(\frac{k_{1} z}{v}\right) \exp \left(-\nu s / k_{2}\right)^{\frac{k_{1} z}{v}} \\
& \approx \exp \left(-(1-\nu) \frac{k_{1} z}{k_{2} v} s\right) \exp \left(\frac{k_{1} z}{v}\right) \frac{1}{1+\nu \frac{k_{1} z}{k_{2} v}}
\end{aligned}
$$

where $\nu \in] 0,1[$ can be seen as a weighting variable which will be discussed later on. This leads to the following transfer function as an approximation of (2)

$$
\exp (-\underbrace{\left(\frac{z}{v}+(1-\nu) \frac{k_{1} z}{k_{2} v}\right)}_{\delta} s) \frac{1}{1+\underbrace{\nu \frac{k_{1} z}{k_{2} v}}_{\tau} s}
$$

As a result, one obtains a delayed first-order step response $\frac{\exp (-\delta s)}{1+\tau s}$ with

$$
\left\{\begin{array}{l}
\tau=\nu \frac{k_{1} z}{k_{2} v} \\
\delta=\frac{z}{v}+(1-\nu) \frac{k_{1} z}{k_{2} v}
\end{array}\right.
$$

The weighting variable $\nu$ relates $\tau$ and $\delta$. Explicitly, we get

$$
\delta=\frac{z}{v}+\frac{k_{1} z}{k_{2} v}-\tau
$$

In an identification standpoint, the next step is to determine a constraint to set the value of $\tau$, which implicitely sets the value of $\nu$.

Inflexion point The second-order derivative of the step response (3) with respect to time is

$$
\begin{gathered}
\frac{\partial^{2}}{\partial t^{2}} T\left(z, t+\frac{z}{v}\right)=-\Upsilon(t) \exp \left(-\frac{k_{1} z}{v}\right) \exp \left(-k_{2} t\right) \times \\
{\left[\left(k_{2}+\frac{1}{t}\right) \sqrt{\frac{m}{t}} \mathrm{I}_{1}(2 \sqrt{m t})-\frac{m}{t} \mathrm{I}_{0}(2 \sqrt{m t})\right]}
\end{gathered}
$$

Using the following asymptotic expansion of Bessel function (Abramowitz and Stegun, 1965)

$$
\left\{\begin{array}{l}
\mathrm{I}_{\nu}(z) \approx \frac{\mathrm{e}^{z}}{\sqrt{2 \pi z}}\left(1-\frac{\mu-1}{8 z}+\frac{(\mu-1)(\mu-9)}{2 !(8 z)^{2}}-\ldots\right) \\
\mu=4 \nu^{2}
\end{array}\right.
$$

at first-order, we get, for $\frac{3}{16 \sqrt{m t}} \ll 1$,

$$
\begin{gathered}
\frac{\partial^{2}}{\partial t^{2}} T\left(z, t+\frac{z}{v}\right) \approx-\Upsilon(t) \exp \left(-\frac{k_{1} z}{v}\right) \exp \left(-k_{2} t\right) \times \\
{\left[\left(k_{2}+\frac{1}{t}\right) \sqrt{\frac{m}{t}} \frac{\mathrm{e}^{2 \sqrt{m t}}}{\sqrt{4 \pi \sqrt{m t}}}-\frac{m}{t} \frac{\mathrm{e}^{2 \sqrt{m t}}}{\sqrt{4 \pi \sqrt{m t}}}\right]}
\end{gathered}
$$

The equation of the inflexion point, of which $t_{I}$ is the unknown abscissa, is given by

$$
\frac{\partial^{2}}{\partial t^{2}} T(z, \underbrace{t_{I^{\prime}}+\frac{z}{v}}_{t_{I}})=0
$$

which leads to

$$
k_{2}+\frac{1}{t_{I^{\prime}}}-\sqrt{\frac{m}{t_{I^{\prime}}}}=0
$$

Then,

$$
t_{I^{\prime}} \approx \frac{k_{1} z}{k_{2} v}\left(\frac{1}{2}+\frac{1}{2} \sqrt{1-\frac{4 v}{k_{1} z}}\right)-\frac{1}{k_{2}}
$$

and assuming $\frac{4 v}{k_{1} z} \ll 1$, we obtain

and finally

$$
t_{I^{\prime}} \approx \frac{k_{1} z}{k_{2} v}
$$

$$
t_{I} \approx \frac{k_{1} z}{k_{2} v}+\frac{z}{v}
$$

Parameters $\tau$ and $\delta \quad$ Note $f$ the delayed first order model step response

$$
f(t)=1-\exp \left(-\frac{t-\delta}{\tau}\right)
$$

Then,

$$
\frac{d}{d t} f(t)=\frac{1}{\tau} \exp \left(-\frac{t-\delta}{\tau}\right)
$$

Let $t_{E}$ be the solution of

$$
f\left(t_{E}\right)=T\left(z, t_{I}\right)
$$

We impose the slope of $f$ at abscissa $t_{E}$ to equal the slope of $T$ at $t_{I}$, i.e.

then, we get

$$
\frac{d}{d t} f\left(t_{E}\right)=\frac{\partial}{\partial t} T\left(z, t_{I}\right)
$$

$$
\tau=\frac{1-T\left(z, t_{I}\right)}{\exp \left(-2 k_{1} z / v\right) k_{2} I_{1}\left(2 k_{1} z / v\right)}
$$

To sum up, with the additional requirement (11), it is possible to write explicit values of $\tau$ and $\delta$

$$
\left\{\begin{array}{l}
\tau=\frac{1-T\left(z, t_{I}\right)}{\exp \left(-2 k_{1} z / v\right) k_{2} I_{1}\left(2 k_{1} z / v\right)} \\
\delta=\frac{z}{v}+\frac{k_{1} z}{k_{2} v}-\frac{1-T\left(z, t_{I}\right)}{\exp \left(-2 k_{1} z / v\right) k_{2} I_{1}\left(2 k_{1} z / v\right)}
\end{array}\right.
$$

Typical identification results are presented in Fig. 4. Two different cases that are representative of real DOC parameter values, as motivated by Lepreux et al. (2008), are reported.

Further approximation of $\tau$ and $\delta$ It has been shown that the choice of the constraint (11) leads to good matching of responses results. Further approximation can be made to prevent evaluation of the Bessel function. In experiment of Fig. 4, we get $\frac{3}{8} \frac{1}{2 k_{1} z / v} \ll 1$ for the two presented cases. Referring to (10), this validates the use an asymptotic expansion of $I_{1}$. We can make the approximation $\mathrm{I}_{1}\left(2 k_{1} z / v\right) \approx \frac{\exp \left(2 k_{1} z / v\right)}{\sqrt{2 \pi 2 k_{1} z / v}}$. Then, we get the following expressions

$$
\left\{\begin{array}{l}
\tau=\frac{1}{k_{2}}\left(1-T\left(z, t_{I}\right)\right) \cdot 2 \cdot \sqrt{\pi} \cdot \sqrt{\frac{k_{1} z}{v}} \\
\delta=\frac{z}{v}+\frac{1}{k_{2}} \frac{k_{1} z}{v}-\frac{1}{k_{2}}\left(1-T\left(z, t_{I}\right)\right) \cdot 2 \cdot \sqrt{\pi} \cdot \sqrt{\frac{k_{1} z}{v}}
\end{array}\right.
$$

It is interesting to note that, considering requirement (11), $\delta$ does not have an hyperbolic behavior with respect to $v$. Experimental results for evolution of $\tau$ and $\delta$ are shown in Fig. 5 (see also Frobert et al. (2009) for more complete results and details about the identification process). Corresponding analytical values are obtained using constant parameters $k_{1}$ and $k_{2}$, and $L_{c}$ a function of $v$ as mentioned in section 2 . 

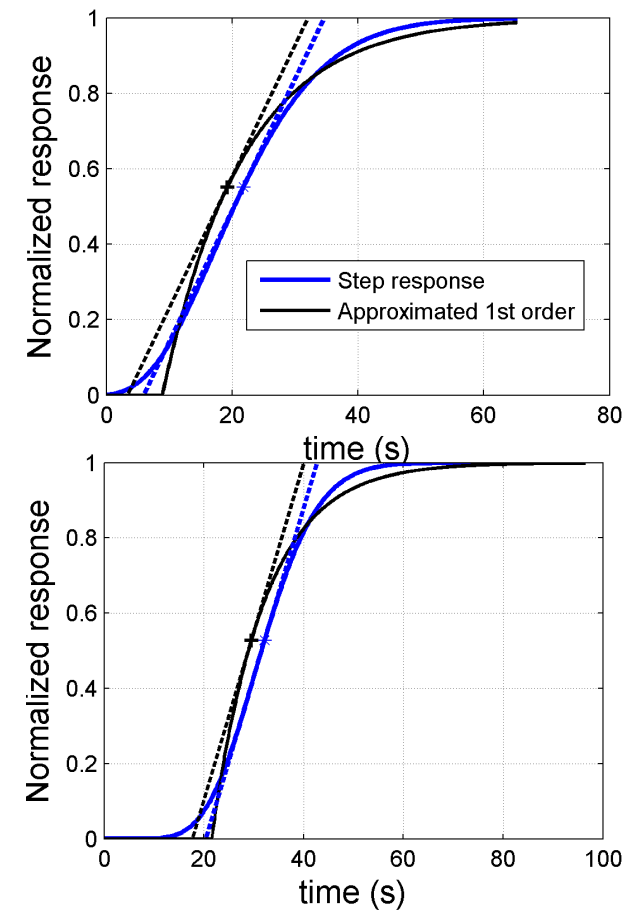

Fig. 4. Matching the DOC response with a first order plus delay model. $k_{1}=400, k_{2}=0.35, v=4$ (up). $k_{1}=1591.09, k_{2}=0.82$, $v=4.597$ (down).
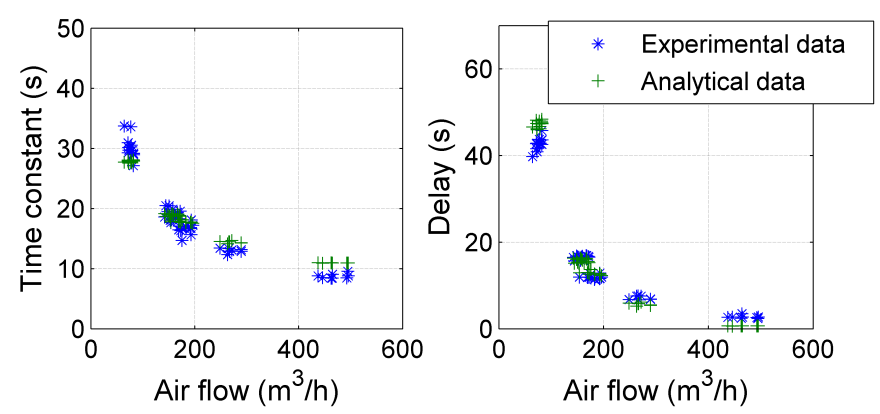

Fig. 5. Experimental evolution of $\delta$ and $\tau$ versus $v$

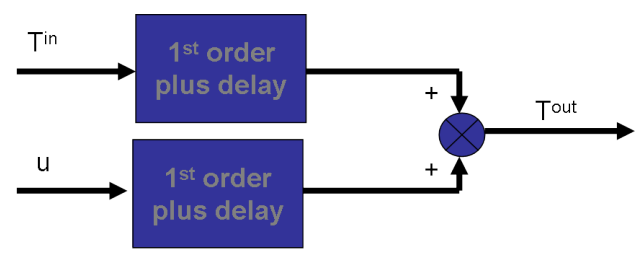

Fig. 6. Scheme of the first order plus delay model

To sum up, the derived model is a combination of two first order plus delay models as shown in Fig. 6. The first one uses $T^{i n}$ as input and $\tau_{T^{i n}}$ and $\delta_{T^{i n}}$ parameters are evaluated by (12) using the whole DOC length. The second one uses $T^{\text {in }}$-equivalent-to$\Psi$ as input, and corresponding $\tau_{u}$ and $\delta_{u}$ are evaluated by the same formula (12) using a part of the DOC length as explained in section 2. In both submodels, $k_{1}$ and $k_{2}$ are constant and equal.

Despite the fact that the proposed method of approximation does not allow to evaluate errors a priori, it is shown in simulations of subsection 4.2 that these methods provide accurate results for real cases of engineering interest such as those of a DOC used in driving conditions.

\section{CONTROLLER PRESENTATION}

\subsection{Control Designs}

Based on the results of section 3, we consider three classical control designs and evaluate their performance. The first two designs are simple PI and PID controllers with a feedforward term as presented in Fig. 7. We use respectively Tavakoli and Fleming (2003) and Tavakoli and Tavakoli (2003) parameters tuning for the PI and the PID controllers. Parameters $\tau$ and $\delta$ are evaluated using (12). The third controller, presented in Fig. 8,

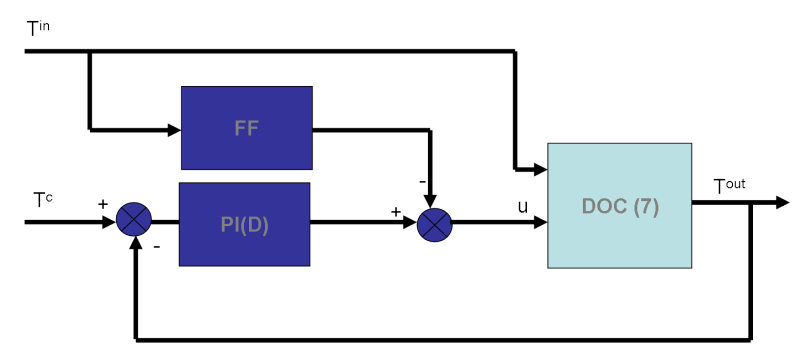

Fig. 7. Control scheme for the PI(D) controller

consists of a Smith controller (Abe and Yamanaka, 2003). For

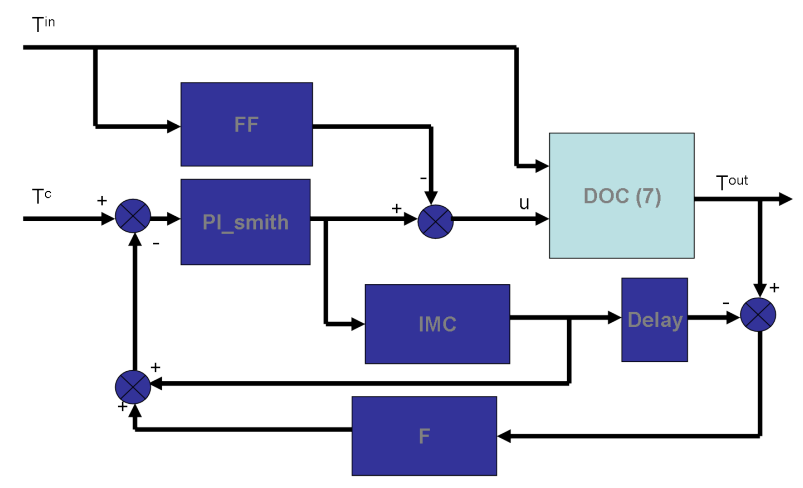

Fig. 8. Control scheme for the Smith controller

the three controllers, the gain $G_{u}$ is calculated using (9). The FF block is dedicated to treating the $T^{i n}$ disturbance. Here, we use a standard feedforward strategy given by (14). Transfer functions and parameters are given as follows

$$
\begin{gathered}
F F=\frac{G_{T^{i n}}}{G_{u}} \frac{\tau_{u} s+1}{\tau_{T^{i n}} s+1} \exp \left(-\left(\delta_{T^{i n}}-\delta_{u}\right) s\right) \\
\text { Delay }=\delta_{u} \\
P I_{\text {Smith }}=\frac{1}{G_{u}}\left(1+\frac{1}{\tau_{u} s}\right) \\
I M C=G_{u} \frac{1}{\tau_{u} s+1} \\
F=\frac{1}{\tau_{f} s+1}
\end{gathered}
$$

where $\tau_{f}$ is the filter constant set to $1 \mathrm{~s}$. The robustness filter $F$ is not primordial here because, thanks to the presented detailed analysis of the DOC equations, delays are well approximated. 


\subsection{Simulation Results}

First, we study the influence of a disturbance step variation. Then, we present control performance during a NEDC driving cycle. Simulation results are shown on the model with source (4) with $k_{1}=870 \mathrm{~s}^{-1}, k_{2}=0.45 \mathrm{~s}^{-1}, L_{c}=0.0305 \mathrm{~m}$, $L=0.0762 \mathrm{~m}$.

Basic performance Fig. 9 compares performance of the three controllers for a setpoint change. Setpoint is risen from 0 to 50 at $t=200$. At the end of the rise, the system is disturbed by an important gas speed variation. These variations are directly linked to driver's power request, they are very fast and cannot be avoided. Although both controllers show similar tracking performance, the Smith controller shows much better disturbance rejection. Similar results are presented in Fig. 10

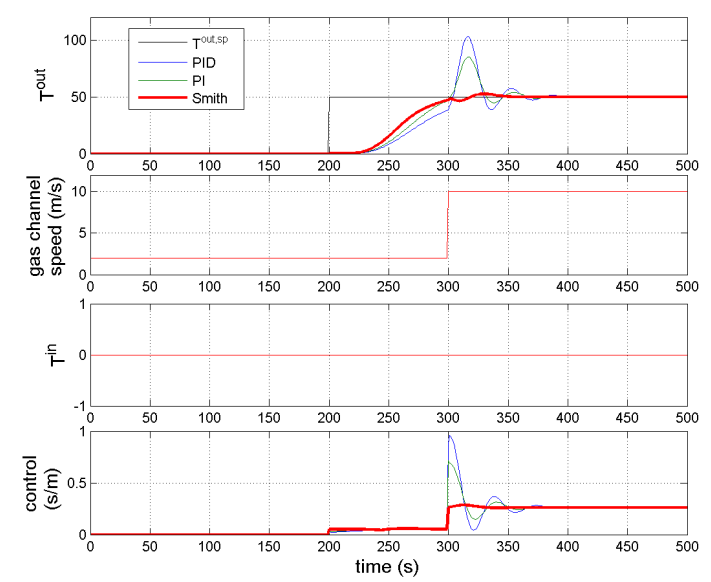

Fig. 9. Step setpoint transition and step v variation for PI, PID, and Smith controllers

with a $20 \%$ error on the $k_{1}$ parameter, implying important delay misestimation. All three proposed controllers are quite robust regarding this error.

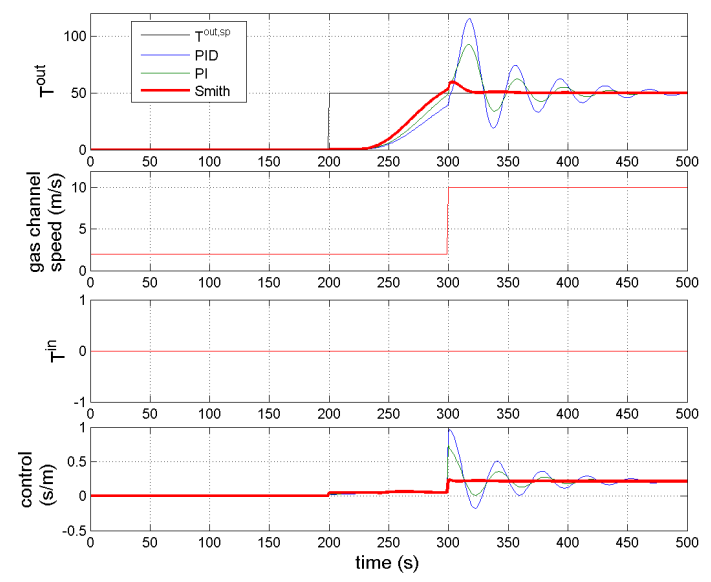

Fig. 10. Step setpoint transition and step v variation for PI, PID, and Smith controllers with a $20 \%$-error on $k_{1}$

Performance on the NEDC cycle The three controllers are now tested on a simulated NEDC cycle. Results are presented in Fig. 11. In this case of a constantly-varying air flow rate, the differences between controllers are very small. Similar results are presented in Fig. 12 with a $20 \%$ error on the $k_{1}$

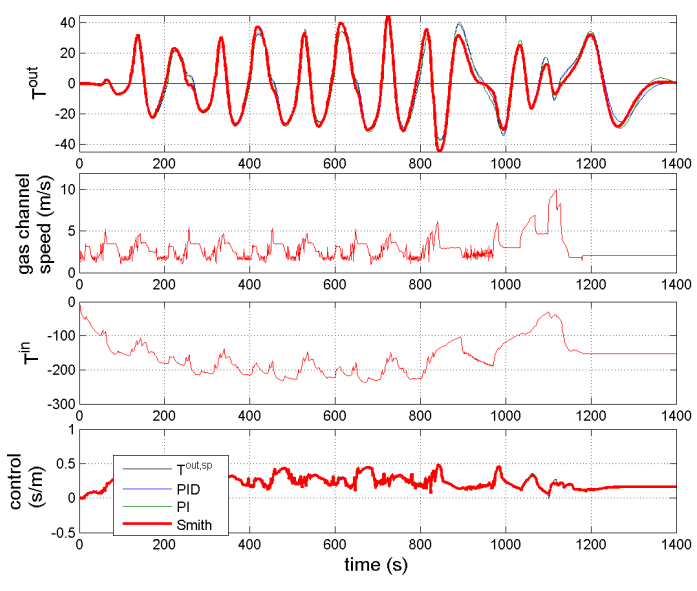

Fig. 11. PI, PID, and Smith controllers on NEDC cycle

parameter. Once again, the presented controllers show good results regarding robustness on this fundamental parameter.

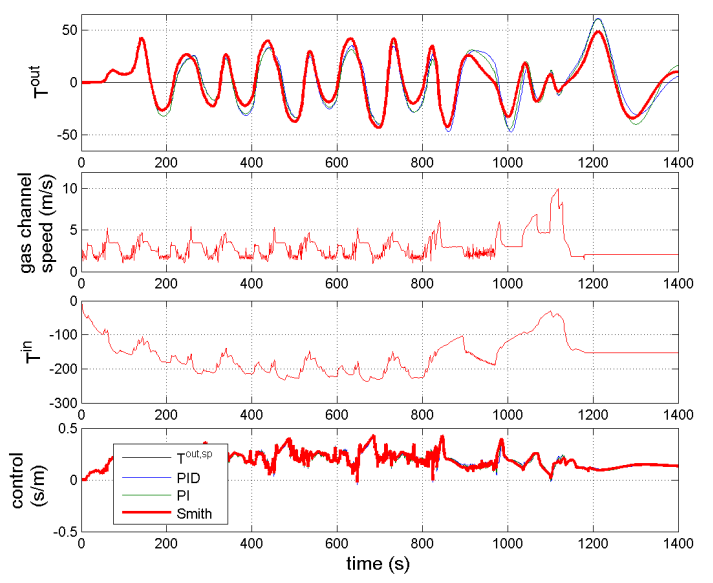

Fig. 12. PI, PID, and Smith controllers on NEDC cycle with a $20 \%$-error on $k_{1}$

Conclusion The presented Smith controller requires more computational effort than the presented PI(D) controllers and shows some advantages in specific cases (setpoint transition, large air flow rate variation). It should be discussed based on further experimentations if it is necessary to use it or not for every specific case of application. It should be noted that its major drawback (lack of robustness toward a misestimation of the delay) has been circumvented thanks to the presented detailed analysis of the DOC equations.

\section{CONCLUSIONS AND FUTURE WORKS}

Control-oriented DOC modeling has been validated with experimental data in a former work (Lepreux et al., 2008). Grounding the current work on these equations and using several steps of approximation lead us, in this paper, to present a simple delayed first-order control-oriented model to approximate the DOC thermal behavior. This model is used as a starting point for control design. Interestingly, presented controllers require no particular tuning effort. They are tested in simulation on NEDC driving cycle. First, a simple PI or PID in which parameters are scheduled using developed approximations, reveal to 
be overreactive in certain specific cases. Alternatively, a Smith design shows good results and turns out to be fairly robust.

The presented experimental results allow a good level of confidence in our model. However, the next step is to present experimental results on the driving cycle using the proposed controllers.

A significant part of performance achieved by controllers presented in this paper, is due to the feedforward treatment of disturbances. To simplify the analysis, the presented controllers use classical feedforward control laws. However, going deeper into analysis, allows to achieve much better results. This feedforward control will be fully detailed in a forthcoming publication.

Being a part of an integrated system in the vehicle, the DOC is subject to whole class of reductants oxidizing onto it. It is worth properly estimating their flow, resulting from in-cylinder combustion, because it represents important disturbances for the DOC outlet temperature controller. This estimator is the subject of future works.

\section{REFERENCES}

Abe, N. and Yamanaka, K. (2003). Smith predictor control and internal model control - a tutorial. In SICE 2003 Annual Conference, volume 2, 1383-1387.

Abramowitz, M. and Stegun, I.A. (1965). Handbook of mathematical functions. Dover Publications.

Achour, L. (2001). Dynamique et contrôle de la régénération d'un filtre à particules Diesel. Ph.D. thesis, École des Mines de Paris.

Angot, A. (1982). Compléments de mathématiques à l'usage des ingénieurs de l'électrotechnique et des télécommunications. Masson, 6ème edition.

Bisset, E. (1984). Mathematical model of the thermal regeneration of a wall-flow monolith diesel particulate filter. Chem. Eng. Sci., 39, 1232-1244.

Depcik, C. and Assanis, D. (2005). One-dimensional automotive catalyst modeling. Prog. Energy Combust. Sci., 31, 308369.

Ecopoint Inc. (2008). DieselNet. Available online: http://www.dieselnet.com/.

Frobert, A., Creff, Y., Lepreux, O., Schmidt, L., and Raux, S. (2009). Generating Thermal Conditions to Regenerate a DPF: Impact of the Reductant on the Performances of Diesel Oxidation Catalysts. In SAE International. Detroit, USA. To appear.

Koltsakis, G. and Stamatelos, A. (1997). Catalytic automotive exhaust aftertreatment. Prog. Energy Combust. Sci., 23, 139.

Lepreux, O., Creff, Y., and Petit, N. (2008). Motion planning for a Diesel Oxidation Catalyst. In Proc. of American Control Conference 2008. Seattle, USA.

Oh, S. and Cavendish, J. (1982). Transients of monolithic catalytic converters: response to step changes in feedstream temperature as related to controlling automobile emissions. Ind. Eng. Chem., 21, 29-37.

Osizik, M. (1977). Basic heat transfer. McGraw-Hill.

Silva, G.J., Datta, A., and Bhattacharyya, S.P. (2005). PID controllers for time-delay systems. Birkhäuser.

Tavakoli, S. and Fleming, P. (2003). Optimal tuning of PI controllers for first order plus dead time/long dead time models using dimensional analysis. In Proc. of the 7th European Control Conference.

Tavakoli, S. and Tavakoli, M. (2003). Optimal tuning of PID controllers for first order plus time delay models using dimensional analysis. In Proc. of the 4th International Conference on Decision and Automation (ICCA'03). Montreal, Canada.

Wolfram Research Inc. (2006). The Wolfram functions site. Available online: http://functions.wolfram.com/. 